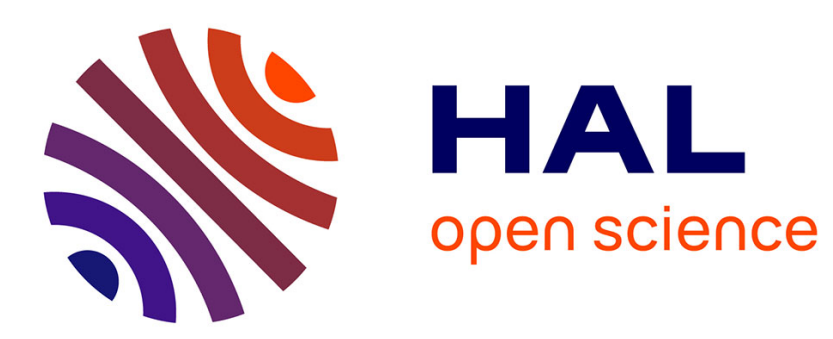

\title{
An Optimization Problem in Distributed Loop Computer Networks
}

Jean-Claude Bermond, G. Illiades, Claudine Peyrat

\section{To cite this version:}

Jean-Claude Bermond, G. Illiades, Claudine Peyrat. An Optimization Problem in Distributed Loop Computer Networks. Annals of the New York Academy of Sciences, 1989, 555 (1 Combinatorial), pp.45-55. 10.1111/j.1749-6632.1989.tb22436.x . hal-02493602

\section{HAL Id: hal-02493602 https://hal.science/hal-02493602}

Submitted on 5 Mar 2020

HAL is a multi-disciplinary open access archive for the deposit and dissemination of scientific research documents, whether they are published or not. The documents may come from teaching and research institutions in France or abroad, or from public or private research centers.
L'archive ouverte pluridisciplinaire HAL, est destinée au dépôt et à la diffusion de documents scientifiques de niveau recherche, publiés ou non, émanant des établissements d'enseignement et de recherche français ou étrangers, des laboratoires publics ou privés. 


\title{
An Optimization Problem in Distributed Loop Computer Networks
}

\author{
J.-C. BERMOND, G. ILLIADES, AND C. PEYRAT \\ Laboratoire de Recherche en Informatique \\ Bâtlment 490 \\ Université Paris-Sud \\ Q1405 Orsay, France
}

\section{INTRODUCTION}

This paper deals with a combinatorial problem arising from studies in distributed and parallel architectures. The interconnection network is the heart of parallel computers, and several topologies have been proposed (see [1], [2], [16]). One of the most used is the loop topology, due to its simplicity [13]; in this case, the network consists of a loop (directed or not), which is modeled by a cycle (directed or not). But the simple loop topology has some disadvantages, in particular, its high vulnerability and low performance. Indeed, the network does not work after the failure of one node or arc (in the directed case) or two nodes or edges (in the undirected case). Furthermore, the diameter of the loop network, which corresponds to the maximum transmission delay, is $n-1$ (in the directed case) or $\left\lfloor\frac{n}{2}\right\rfloor$ (in the undirected case).

A simple way to improve the performance of the network is to increase its connectivity and decrease is diameter. That can be done by adding links. However, for cost and design reasons (in particular there can only be a limited number of links leaving a node), one wanis to add only a few links in each node. Furthermore, to maintain easy roulings and implement distributed algorithms, the addition of these links must be done in a symmetric (or homogeneous) way.

Different "loop networks" (i.e., 'networks representable by Hamiltonian graphs or digraphs) have been proposed. We refer the reader to the recent survey of Bermond, Comellas, and Hsu [4] and its references.

Note that if one only wants to find a network with a limited degree and a good diameter, one can choose the de Bruijn or Kautz digraphs. Indeed these digraphs have outdegree and indegree $d, d^{D}$ or $d^{\mathbb{D}}+d^{\mathbb{D}-1}$ vertices, and diameter $D$. They can be considered as iterated line digraphs of the complete digraphs with of without loops. Therefore, being line digraphs of Eulerian digraphs, these graphs are Hamiltonian. Generalizations of these digraphs for any value of $n$ have been considered by different authors, and recently Du, Hsu, Hwang, Zhang [8] and Du and Hwang [9] proved that these generalized digraphs are Hamiltonian except when the out-and indegree is 2 and $n$ is odd. However, in the case $d=2$, a class of networks obtained by modifying the generalized Kautz digraphs has been proposed by Du, Hsu, and Hwang [7]. In summary, there exist, for any $d$ and $n$, digraphs of order $n$, indegree 
and outdegree $d$, which are Hamiltonian and have a diameter roughly equal to $\left\lceil\log _{\alpha} n\right\rceil$. These digraphs haye the best possible connectivity (mainly $d$ or $d-1$ ). For results on the vulnerability of these digraphs, see the survey of Bermond, Homobono, and Peyrat [3].

If one also wants some strong symmetry properties, one can consider multiplestep digraphs or graphs. Let us denote by $G\left(n ; s_{1}, s_{2}, \ldots, s_{k}\right)$ the $k$ fixed-step digraph, with $n$ vertices labeled with the integers modulo $n$, and such that there is an arc from node $i$ lo the $k$ nodes $i+s_{1}, i+s_{2}, \ldots, i+s_{k}$. The problem of determining the minimum of the diameter of these $k$ fixed-step digraphs among all the possible choices for $s_{1}, s_{2}, \ldots, s_{k}$ is far from being solved, although important results have been obtained, particularly when $s_{1}$ is fixed to 1 (see the survey in [4]). For example, in the case of double fixed-step digraphs $G\left(n ; s_{1}, s_{2}\right)$ the diameter is at least $\lceil\sqrt{3 n}\rceil$ -2 (see [15]). Infinite families of values of $n$ for which this bound may be achieved have been exhibited (Erdös and Hsu [10], Fiol, Yebra, Alegre, and Valero [11], Hwang and Xu [12]), but the exact value of the minimum diameter of the double fired-step digraphs is still generally unknown. Mean distance and vulnerability problems have also been considered.

Here, we restrict our attention to the undirected case where the $k$ fixed-step graph $G\left(3 ; \pm s_{1}, \pm s_{2}, \ldots, \pm s_{k}\right)$ is the graph such that there is an edge between the node $i$ and the nodes $i \pm s_{1}, \ldots, i \pm s_{k}$. We deal mainly with the case of double fixed-step graphs. We determine the exact yalue of the minimum diameter for all values of $n$. We also determine the minimum mean distance, and study the vulnerability of these graphs. The problem of determining their diameter was first considered by Wong and Coppersmith [15]. If the undirected case has received less atiention than digraphs in the context of loop networks, they have, however, been well studied in graph theory as "circulant graphs" or particular cases of "Cayley graphs."

The results were obtained in 1984 and preserited at the conference in New York (June 1985). At this conference, Hsu informed us that the results concerning the diameter have also been independently obtained by Boesch and Wang [6]. They have also been independently obtained by Yebra, Fiol, Morillo, and Alegre [17].

\section{DOUBLF-LOOP GRAPHS}

Here we consider undirected double fixed-step loop networks. Such networks are modeled by undirected graphs with $n$ vertices labeled with the integers modulo $n$. Let $a$ and $b$ be two integers between $I$ and $(n-1) / 2$. Then cvery vertex $i$ is joined by an edge to the vertices $i t a$ and $i \pm b$ (modulo $n$ ).

We will call such a graph a "double-loop graph" and denote it by DLG $(n ; a, b)$. Let us note that these graphs are also known as circulant graphs and can be seen as Cayley graphs associated to the group of the integers modulo rs. Fugure 1 shows DLG $(11 ; 2,3)$.

'The following two propositions are casy:

Protosition $1: \operatorname{DLG}(n ; a, b)$ is regular of degree 4 . 


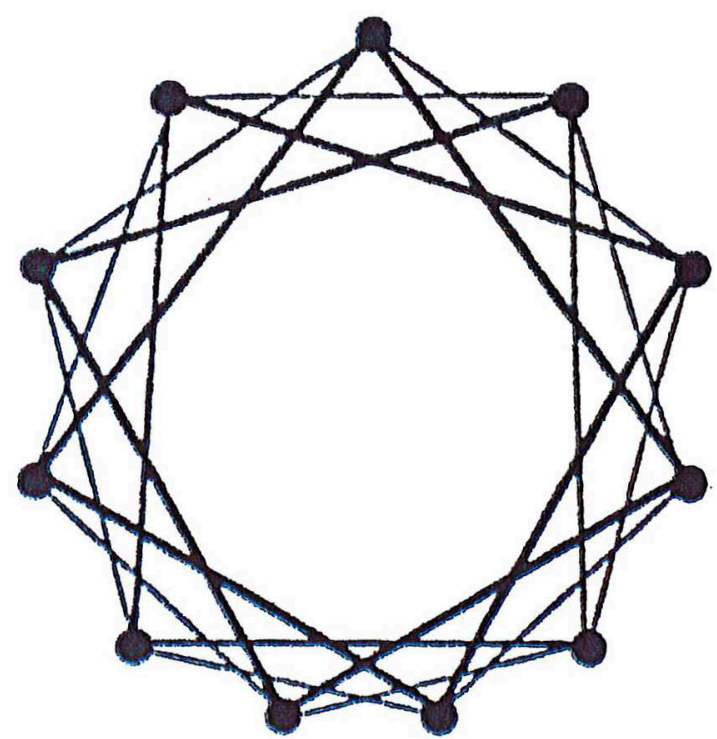

FIGURE 1 。

Profosition 2: DLG $(n ; a, b)$ is connected if and only if $\operatorname{gcd}(a, b, n)=1$ (where $\operatorname{gcd}(a, b, n)$ denotes the greatest common divisor of $a, b$, and $n)$.

The following theorem is a particular case of a theorem obtained independently by different authors saying that any connected Cayley graph on an Abelian group is Hamiltonian (see the survey of Witte and Gallian [14]).

PROPOSITION 3 [sec 14]: If $\operatorname{gcd}(a, b, n)=1$, then DLG $(n ; a, b)$ is Hamiltonian.

That shows that if $\operatorname{DLG}(n ; a, b)$ is connected, then it is a loop network. In fact, a stronger result has been proved by Bermond, Favaron, and Maheo [5]. It follows that these graphs can be seen as the union of two edge disjoint loops and that each loop can be used for different purposes of transmission of the messages. This result was conjectured in an earlier version of this paper and justifies the terminology "double-loop graphs."

Proposition $4[5]$ : If $\operatorname{gcd}(a, b, n)=1$, then DLG $(n ; a, b)$ can be decomposed into two Hamiltonian cycles.

\section{DIAMETER}

The distance $d(x, y)$ between two vertices $x$ and $y$ is the length of a shortest $x-y$ path. The diameter $D$ of a graph is the maximum distance over all the pairs of vertices.

$$
D=\max _{(x, y) \in V x, y} d(x, y)
$$


The mean distance $\bar{D}$ is equal to

$$
\bar{D}=\frac{1}{m(n-1)} \sum_{(x, y) \in V \times V} d(x, y) .
$$

$A$ double-loop graph being vertex transitive, if the vertices are labeled by $0,1, \ldots$, 16-1:

$$
D=\max _{1 \leq i \leq A-1} d(0, i)
$$

and

$$
\bar{D}=\frac{1}{n-1} \sum_{1 \leq i \leq n-1} d(0, i)
$$

The optimization problem considered in the Introduction consists in finding $a$ and $b$ that minimize $D$ and $\bar{D}$, for a given $n$. This problem was already considered by Wong and Coppersmith [15] in the case $a=1$. In the general case it has also been considered by Yebra, Fiol, Morillo, and Alegre [17] and by Boesch and Wang [6], who studied circulant graphs with minimum diameter.

Following Fiol, Yebra, Alegre and Valero [11] and Yebra, Fiol, Morillo, and Alegre [17], we can visualize the problem in a geometric manner. We will represent the edge $(i, i+a)$ by a horizontal segment and the edge $(i, i+b)$ by a vertical one. The distance between two points is obtained by adding the number of horizontal and vertical segments. FIGURE 2 shows the vertices obtained at a distance of at most $k$ of the vertex 0 . In this geometric context, the problem consists in finding a tile consisting of $n$ unit squares that tessellates periodically the plane and to find $a$ and $b$ such that the $n$ unit squares of the tile are numbered from 0 to $n-1$ (see FIG. 3 for such a realization with $n=13, a=2, b=3$ ).

First let us consider the related optimization problem of finding the maximum order of a double-loop graph of given diameter $k$.

The first theorem can be found in [6], [15], and [17]. Here we repeat the proof, in order to be complete.

ThEorem 1: The maximum order of a double-loop graph of diameter $k$ is $2 k^{2}+2 k+1$.

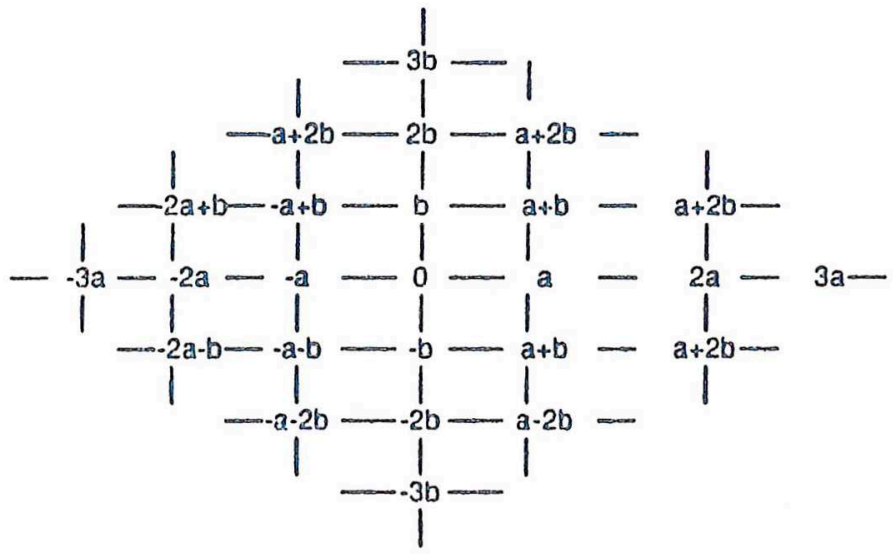

PIGURM 2. 


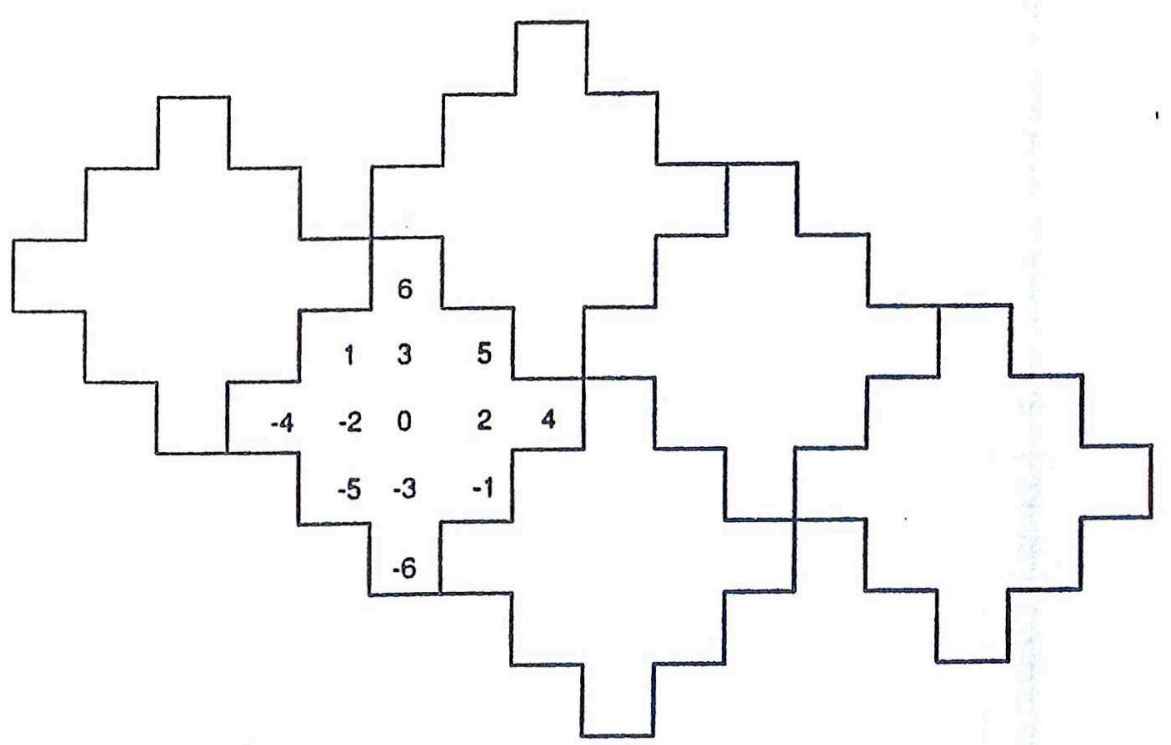

FICURE 3.

Proof: The yertices at distance $j$ from the vertex 0 are $\pm j a, \ldots, \pm(j-i) a \pm i b$, $\ldots . \pm j b$ (with $0 \leq i \leq j$ ), and the maximum number of such vertices is $4 j$. Therefore, the maximum number of vertices of the graph is at most

$$
1+\sum_{j \leq k} 4 j=2 k^{2}+2 k+1
$$

The following theorem shows that the bound is attained (the result has also been obtained independently by Boesch and Wang [6] and Yebra, Fiol, Morillo, and Alegre [17]). We furthermore determine all the possible sets of values for $a$ and $b$.

THEOREM 2: There exists a DLG $\left(2 k^{2}+2 k+1 ; a, b\right)$ of diameter $k$ if and only if $a \equiv k p$ and $b \equiv(k+1) p$, where $p$ is any integer less than $n$ that is relatively prime with $n$.

Proof: "The fact that the bound is attained with $a=k, b=k+1$ follows either from arithmetical conditions or from a geometrical point of view: for example, one can check that all the vertices at distance at most $k$ from 0 are all the integers between $-k(k+1)$ and $k(k+1)$, which, modulo $2 k^{2}+2 k+1$, are exactly all the elements. Geometrically, one can see that the integers in a tile are ranged enumeratively in a diagonal SE-NW, and that this tile (in the form of a cross) tessellates the plane.

The determination of all the values of $a$ and $b$ such that DLG $\left(2 k^{2}+2 k+1 ; a, b\right)$ has diameter $k$ is implicitly contained in [17] and follows from geometrical considerations. Indeed we must have:

$$
\begin{aligned}
& (k+1) a-k b \equiv 0[\bmod n] \\
& k a+(k+1) b \equiv 0[\bmod n] .
\end{aligned}
$$

As the determinant is congruent to 0 modulo $n$, this system has nontrivial solutions that are of the form $a \equiv k p$ and $b \equiv(k+1) p$, where $p$ and $n$ are relatively prime to assure that the graph is connected. 


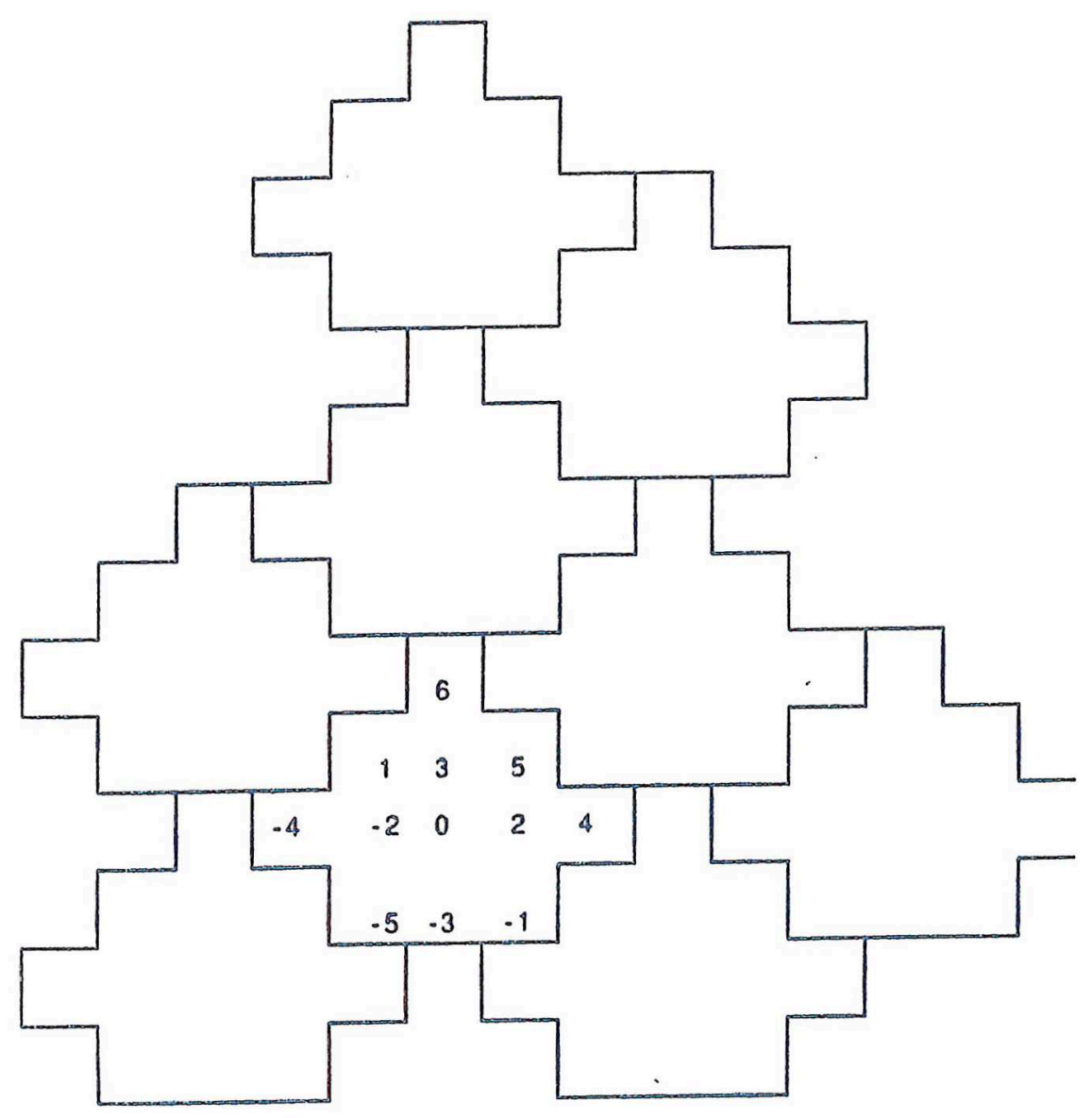

RICURE 4 。

Now let us come back to the original problem of determining the minimum diameter of DLG $(n ; a, b)$. The following theorem has also been obtained by Boesch and Wang [6]. It says that the minimum diameter is

$$
k_{0}=\left\lfloor\frac{1+\sqrt{2 n-3}}{2}\right\rfloor=\left\lceil\frac{-1+\sqrt{2 n-1}}{2}\right\rceil \text {. }
$$

THEOREM 3: If $2(k-1)^{2}+2(k-1)+1<n \leq 2 k^{2}+2 k+1$, then the minimum diameter of DLG $(n ; a, b)$ is $k$, and the bound is achieved with $a=k$ and $b=k+1$.

Proof: By Theorem 1 the diameter is at least $k$. Furthermore, as we have seen in the proof of Theorem 1, the vertices at a distance of at most $k$ are all the integers beiween $-k(k+1)$ and $k(k+1)$, and therefore all the integers modulo $n$ as $n \leq 2 k^{2}+2 k+1$. This fact is also illustrated geometrically. Indeed the tiles obtained by deleting unit squares on the diagonal also tessellate the plane (see FIGS. 4 and 5 for $n=12,11$ ).

REMARS: The determination of all the values $a$ and $b$ such that the diameter of DLG $(n ; a, b)$ is minimum for a given $n$ in the preceding interval is an open problem. 


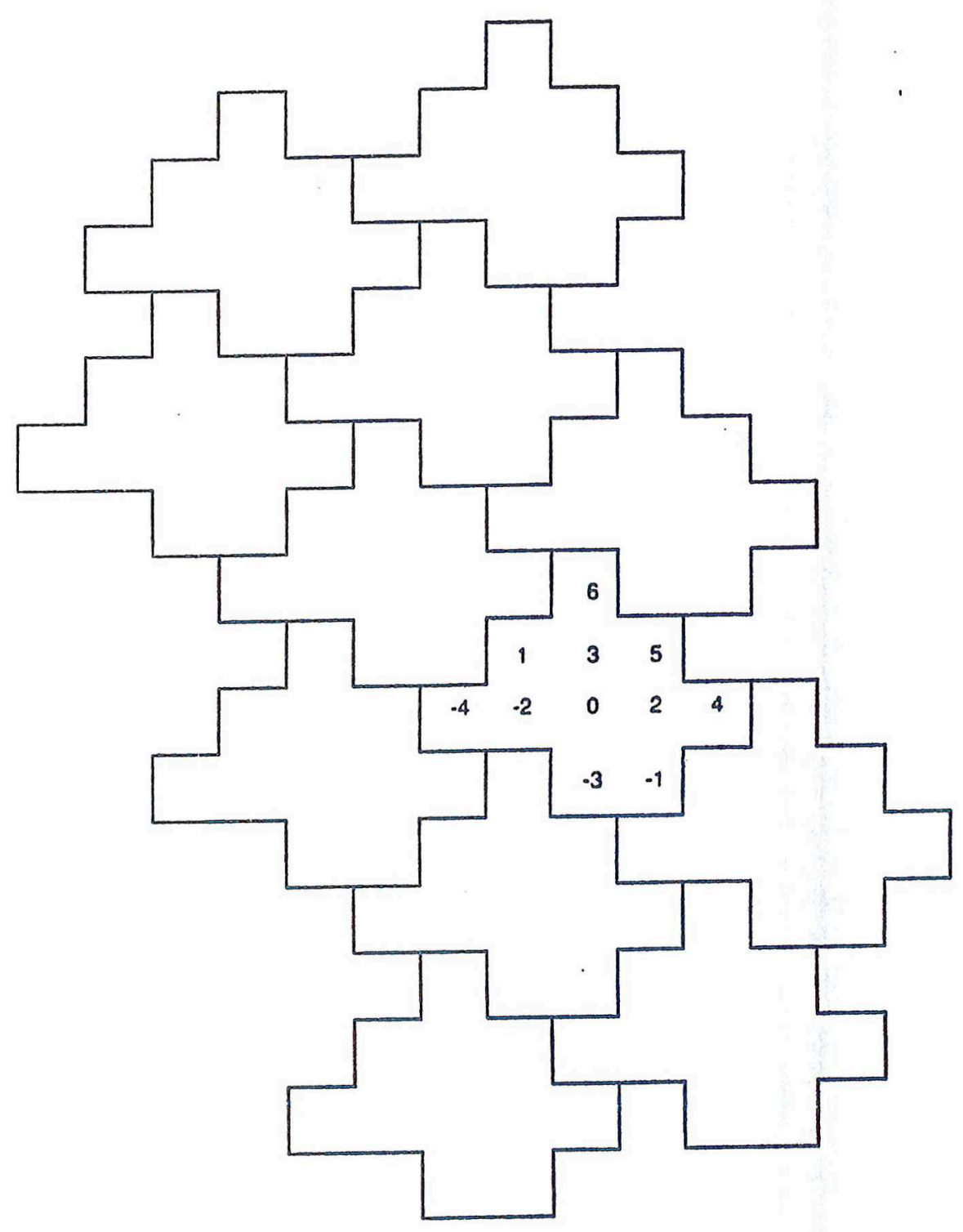

FIGURE 5.

At present the minimum diameter of $\operatorname{DLG}(n ; 1, b)$ is not known. This was the original problem of Wong and Coppersmith [15]. Partial results have recently been obtained by Bermond and Tzvieli [5], Du, Hsu, Li, and Xu [8], and Tavieli [13]. Another set of values that will be useful to compute the mean distance is given in the following theorem.

THEOREM 4: If $2(k-1)^{2}+2(k-1)+1<n \leq 2 k^{2}+1$, then the diameter of DLG $(n ; k-1, k)$ is $k$.

Proof: By 'Theorem 3, all the vertices $i$ such that $|i| \leq k^{2}-k$ are at a distance of at most $k-1$. It suffices to prove that the remaining vertices are at a distance of at most $k$. That follows from the fact that a vertex $i$ is adjacent to vertices $i \pm k$, and therefore the neighborhoods of the vertices $i$ such that $|i| \leq k^{2}-k$ contain all the vertices $j$ such that $|j| \leq k^{2}$. 


\section{MEAN DISTANCE}

THEOREM 5: The mean distance of a double-loop graph of order $n$ is at least $\bar{k}_{0}$ swith

$$
\bar{k}_{0}=k_{0}\left(1-\frac{2 k_{0}^{2}-2}{3(n-1)}\right)
$$

พhinere

$$
k_{0}=\left\lfloor\frac{1+\sqrt{2 n-3}}{2}\right\rfloor
$$

This bound is attained for every $n$ by considering DLG( $(n ; a, b)$ with $b=a+1$ and $a=k_{0}$ or $k_{0}-1$.

Proof: Recall that $(n-1) \bar{D}=\sum_{i \leq n-1} d(0, i)$ and that we have at most $4 j$ vertices at distance $j$ from the vertex 0 . Therefore:

$$
\begin{aligned}
& (n-1) \bar{J} \geq 4 \sum_{j=1}^{k_{0}-1} j^{2}+k_{0}\left(n-\left(2 k_{0}^{2}-2 k_{0}+1\right)\right) \\
& (n-1) \bar{D} \geq(n-1) k_{0}-\frac{2 k_{0}^{3}-2 k_{0}}{3}
\end{aligned}
$$

therefore $\bar{D} \geq \bar{k}_{0}$. "\%o show that the bound is attained, we will distinguish two (nondisjoint) cases:

Case 1: $n \geq 2 k_{0}^{2}-1$. Consider DLG( $\left.n ; k_{0}, k_{0}+1\right)$. The vertices $i$ such that $\mid$ ii $\mid \geq k_{0}^{2}$ are at a distance of at least $k_{0}$ and therefore (by Theorem 2) $k_{0}$ from the vertex 0 . Therefore, as $s \geq 2 k_{0}^{2}-1$ the distribution of the vertices at distance $j$, with $j<k_{0}$, from the vertex 0 is the same as in DLG $\left(2 k_{0}^{2}+2 k_{0}+1 ; k_{0}, k_{0}+1\right)$ and we have exactly $4 j$ vertices at distance $j$ for $j<k_{0}$ and the remaining vertices at distance $b_{0}$, which is the optimal configuration.

Case 2: $n \leq 2 k_{0}^{2}+1$. Consider DLG $\left(n ; k_{0}-1, k_{0}\right)$. By Theorem 1 , all the vertices $i$ such that $|i| \leq k_{0}^{2}-k_{0}$ are at at distance of at most $k_{0}-1$, and there are

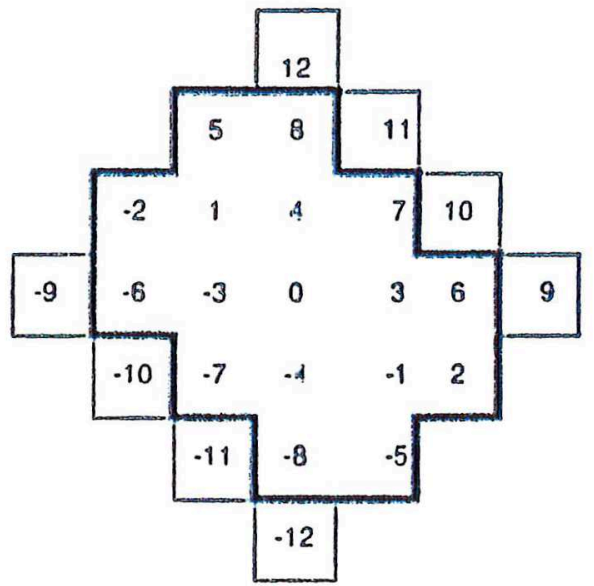

FIGURE 6. 


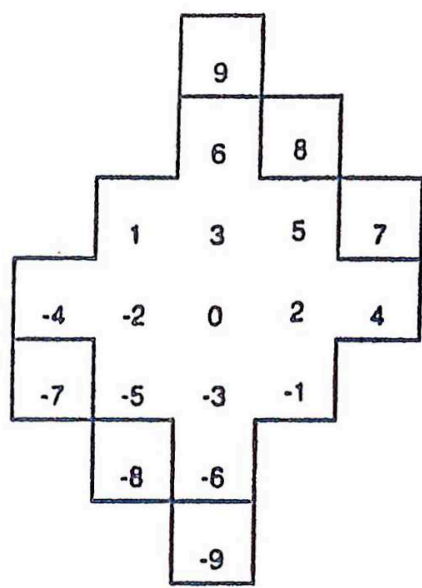

FIGURE 7 .

exactly $4 j$ vertices at distance $j$ from the vertex 0 with $j \leq k_{0}-1$. The other vertices are at distance $k_{0}$ by 'Theorem 4.

REMARF 1: Asymptotically the mean distance is $\sqrt{2 n} / 3$.

REMARK 2: Geometrically the proof means that if $n>2 k_{0}^{2}-1$, the tile corresponding to DLG $\left(n ; k_{0}, k_{0}+1\right)$ is obtained by deleting unit squares of the extremal diagonals (that correspond to vertices at distance $\left.k_{0}\right)$ of $\mathrm{DLG}\left(2 k_{0}^{2}+2 k_{0}+1 ; k_{0}\right.$, $k_{0}+1$ ) (FIG. $6, n=17$ ). Similarly for $n \leq 2 k_{0}^{2}+1$, the tile is obtained by adding unit squares in the neighborhood of the extremal diagonals of the tile associated to DLG $\left(2 k_{0}^{2}-2 k_{0}+1 ; k_{0}-1, k_{0}\right)$ (see FIG. $7, n=19$ ).

\section{ROUTING}

The routing in the graph DLG $(n ; k, k+1)$ is very simple. To find a shortest path between $i$ and $j$, it suffices to determine $p$ and $q$ such that $j-i=p k+q(k+1)$ with $p, q$ integers and $|p|+|q|$ the smallest possible. Then there exists $\left(\begin{array}{c}|p|+|q| \\ |p|\end{array}\right)$ shortest paths from $i$ to $j$ consisting of $|p|$ steps of the kind $k$, and $|q|$ steps of the kind $k+1$.

\section{VULNERABLLTTY}

Boesch and Wang $\left[6\right.$, theorem 5] proved that a $\operatorname{DLG}\left(n ; k_{0}, k_{0}+1\right)$ has connectivity 4. Here we study the effect of the deletion of a vertex (or an edge) on the diameter.

THEOREM 6: "The diameter of the graph obtained by deleting one vertex or one edge in DLG $\left(n ; k_{0}, k_{0}+1\right)$ is at most $k_{0}+1$.

proof: As we have seen for the routing, there are $\left(\begin{array}{c}|p|+|q| \\ |p|\end{array}\right)$ shortest paths between yertices $i$ and $j$. Therefore, after deletion of one vertex $s$ or one edge $e$, the 
distance can increase only if all the shortest paths contained this vertex or edge. This implies that $p=0$ or $q=0$, and that the vertex (or edge) deleted is in the path. Furthermore, if the yertes $s$ deleted belongs to such a path $s-h, s, s+h$ (with $|h|=k_{0}$ or $\left.k_{0}+1\right)$, we can replace it by the subpath $s-h_{,} s-h+h^{\prime} s+h^{\prime}$, $s+h^{\prime}+h, s+h$, with $h^{\prime}=k_{0}$ or $k_{0}+1$ according $|h|=k_{0}$ or $k_{0}+1$. Similarly, we can replace the edge $s, s+h$ by the subpath $s, s+h^{\prime}, s+h^{\prime}+h_{0} s+h$. In any case, the distance can increase at most by 2 . Therefore, to prove that the diameter of DLG $\left(k_{0}, k_{0}+1\right)$ is at most $k_{0}+1$, it suffices to prove that there exists a path of length $k_{0}+1$ between two vertices $i$ and $j$ such that $j-i= \pm k_{0}^{2}$ or $\pm\left(k_{0}^{2}+k_{0}\right)$. If $j-i=\varepsilon k_{0}^{2}$, such a path consists in $k_{0}$ edges with step $\varepsilon\left(k_{0}+1\right)$ and one edge with step $-\varepsilon k_{0}$; indeed, $k_{0}^{2}=k_{0}\left(k_{0}+1\right)-k_{0}$. Similarly, if $j-i=\varepsilon\left(k_{0}^{2}+k\right)$, it suffices to take $k_{0}+1$ edges with step ek.

REMARK: The diameter can be $k_{0}+1$ or $k_{0}$. For example, in DLG(18;3,4), there are two disjoints paths of length $\leq 3$ between any pair of vertices, but in DLG $(19 ; 3,4)$, there is only one path of length $\leq 3$ between 0 and 6 .

\section{OPEN PROBLEM}

One can ask for similar problems with multiple loops instead of double loops. But, even in the case of three loops there exists no perfect optimal solution. The determination of the minimum diameter might be a difficult problem. In that case, it follows from a result of Wong and Coppersmith [15] (see also [0]) that the minimum diametci $d(n)$ of a triple fixed loop graph $G\left(n ; \pm a_{2} \pm b, \pm c\right)$ satisfies $f(n) \leq d(n) \leq g(n)$, where $f(n)$ is of order $(3 n / 4)^{1 / 3}$ and $g(n)$ is of order $(27 n / 8)^{1 / 3}$. Recently, Peyrat has shown that $g(n)$ may be reduced to a function of order $(9 n / 8)^{1 / 3}$.

Recent results have recently been obtained on the determination of the minimum diameter of DLG $(n ; 1, b)$ by Bermond and Tzvieli [5], Du, Hsu, Li, and $\mathrm{Xu}$ [8], and Tryieli [13].]

\section{REFERENCES}

1. HaROND, J.C. J. BOND, M. PAOL \& C. PEYRAT. 1983. Graphs and interconnection neto works: Diameter and vulnerability. In Pros. Ninth British Combinatorial Conference. London Mathematical Society, Lecture Note Series 82: 1-30.

2. PARMOND, J.C., C. DhLORM! \& J.-J. QuisquATRR. 1986. Strategies for interconnection neto works: Some methods from graph theory. J. Parallel Distrib. Comput. 3: 433-449.

3. Berrond, J.-C. N. HOMOmoNo \&. C. PEYRAT. 1986. Large fault tolerant interconnection networks. Is Proc. First Japan International Conference on Graph 'Theory and Applications, Hakone, Japan; also, accepted for publication in Graphs Comb.

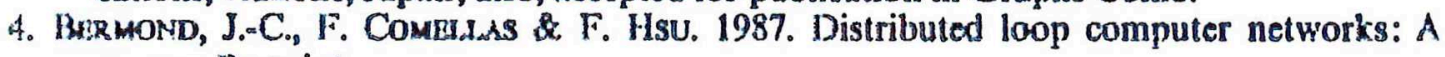
survey. Preprint.

5. BXeRHOND, J.-C., O. FAYARON M. Marizo. 1988. Hamiltonian decomposition of Cayley graphs of degree four. J. Comb. 'Theory, Ser. 円. In press; Bermond, J.-C. \&. D. Tzvieu. Minimal diameter double-loop networks: Part II: Dense optimal families. Submitted for publication in Networks.

6. BOEscH, F. T. \& J. K. WANG. 1985. Reliable circulant networks with minimum transmission delay. ISER 'Trans. Circuits Syst. CAS-32: 1286-1291. 
7. DU, D. Z., D. F. Hsu \& F. K. Hwang. 1985. Doubly-linked ring networks. IEEE Trans. Comput. 34: 853-855.

8. Du, D. Z. D. F. Hsu, F. K. Hwang \& X. M. Zmang. 1988. The hamiltonian property of generalized de Bruijn graphs. J. Comb. Theory. In press; DU, D. Z., D. F. Hsu, Q. LI \& Q. XU. 1988. A combinatorial problem related to distributed loop networks. Networks. In press.

9. Du, D. Z. \& F. K. Hwang. 1987. Generalized de Bruijn Graphs. Preprint.

10. ERDö́s, 1. \& D. F. Hsu. 1988. Distributed loop networks with minimum transmission delay. Theor. Comput. Sci. In press.

11. FlOL, M. A., J. L. A. Yebra, I. Alegre \& M. VAlero. 1987. A discrete optimization problem in local networks and data alignment. IEEE Trans. Comput. C-36: 702-713.

12. HWANG, F. K. \& Y. H. XU. 1987. Double loop networks with minimum delay. Discrete Math. 66: 109-118.

13. LU, M. T. 1978. Distributed loop computer networks. In Advances in Computers. Vol. 17: 163-221. Academic Press. New York; Tzvisu, D. 1988. Double loop networks: Optimal and suboptimal families. Preprint.

14. WITTE, D. \& J. A. GALLIAN. 1984. A survey: Hamilton cycles in Cayley graphs. Discrete Math. 51:293-304.

15. WONG, C. K. \& D. Coppersmith. 1974. A combinatorial problem related to multimodule memory organizations. J. ACM. 21(3): 392-402.

16. WU, C. L. \& T. Y. Feng. 1984. Tutorial: Interconnection networks for parallel and distributed processing. IEEE Computer Society. New York.

17. Yeara, J. L. A. M. A. Fiol, P. Morrllo \& I. AlfGre. 1985. The diameter of undirected graphs associated to plane tessellations. In Proc. Tenth British Combinatorial Conference, Ars Combinatoria 20B: 159-171. 